\title{
A Context-Aware Inter-organizational Collaboration Model Applied to International Trade
}

\author{
Jie Jiang, Virginia Dignum, Yao-Hua Tan, and Sietse Overbeek \\ Faculty of Technology, Policy and Management, Delft University of Technology, \\ 2628 BX Delft, The Netherlands \\ \{jie.jiang,m.v.dignum,y.tan, s.j.overbeek\}@tudelft.nl
}

\begin{abstract}
In international trade, there are a number of aspects that influence the interactive relationships between business organizations and governmental organizations, which makes it difficult to regulate the business processes in an integrated way. Modeling such kinds of organizational interactions requires a mechanism to differentiate interactive environments and elaborate regulations according to their characteristics. For this purpose, a context-aware interorganizational modeling approach is proposed in this paper. The approach analyzes organizational interactions through three phases from abstract to concrete: (1) general specifications which describe organizations in terms of atomic roles with intellectual objectives, (2) contextual specifications which extend general specifications by applying contexts to derive composite roles with details on how to accomplish the objectives, and (3) operational specifications which construct a set of complete models of an interorganizational collaboration by assembling contextual specifications according to the run-time environment. An example consisting of two scenarios of direct control and self-regulation in international trade is used to illustrate our model.
\end{abstract}

Keywords: Context, international trade, OperA, organization modeling, roles.

\section{Introduction}

With the rapid development of international trade, strategic alliances, collaborative commerce, virtual corporations, and value chain integration of multiple organizations are established to achieve better performance [1]. Collaborative organizations are involved in the value chain to accomplish not only their own goals but also the cooperative goals. On the one hand, business organizations try to operate as efficiently as possible. On the other hand, governmental organizations have to perform tasks in the area of security to regulate business performance. Governmental control and regulation of complex multi-organization alliances is not only timeconsuming but also costs a great deal of human resources. Hence, interactions between business and governmental organizations is changing from monolithic control by regulatory authorities to distributed environments where private enterprises are free to regulate their affairs within boundaries set by the regulatory authorities. The former way of controlling is called direct control and the latter is named 
self-regulation. In order to determine the effects and possibilities of different approaches for direct control and self-regulation, a careful analysis is required to make sure that integrated business processes are performed in a secure and smooth way. To this end, we propose a framework that enables modeling and comparisons between different inter-organizational collaborative approaches. A large number of aspects influence the regulative relationships between business and governmental organizations, such as:

- $\quad$ Diversity of business types: food, clothes, electronic devices, etc.

- $\quad$ Diversity of regulation policies: $\mathrm{AEO}^{1}, \mathrm{C}_{-} \mathrm{TPAT}^{2}$, etc.

- Diversity of partnerships: long-term, short-term, etc.

Combinations of these factors result in different relations between governments and businesses due to the different policies applicable in each case. For example, the safety requirements for an AEO certificate are interpreted quite differently for a company that is exporting dairy products (risk of food safety) than for a company that is exporting scrap metal (risk of hidden bombs). Even though at an abstract level, regulative relations between governments and businesses can be modeled in the same way, the diversity of specific contexts and their characteristics leads to an explosion of interactive models (i.e., models are case specific and not re-usable), which makes it difficult for both business and governmental organizations to articulate their interactions. Context-aware applications provide potential solutions to this problem as they look at who's, where's, when's and what's to determine why the situation is occurring [2].

Moreover, the individual characteristics of actors in the interactions need to be further detailed to make sure that they can fit the requirements and restrictions of both businesses and governments. Therefore, the main objective of this paper is to describe how to model organizational interactions following a contextual refinement process, i.e., from abstract to concrete. This enables each partner in the supply chain to specify their responsibilities precisely and avoid unpredictable failures such as unmatchable information, misbehaving, etc.

Business organizations and governmental organizations interact with each other within a set of regulations, which can be described as a Multi-Agent Systems (MAS) where multiple intelligent agents interact within a set of norms. In a given MAS, cooperation between agents that possess diverse knowledge and capabilities facilitate the achievement of global goals that cannot be achieved by a single agent. MASs have been shown to be highly appropriate for the modeling of open, distributed, and heterogeneous systems [3]. OperA [4], being an agent-based modeling framework, has provided a basis for modeling multi-organization interactions. Therefore, in this paper we extend its architecture and present a framework that not only involves the notion of context but also supports a contextual refinement modeling process. The remainder of this paper is organized as follows. In section 2, the background and

\footnotetext{
${ }^{1}$ The Authorized Economic Operator (AEO) is a European-wide customs initiative that aims to secure the supply chain while at the same time reducing the administrative burden for actors through the use of self-regulation.

${ }^{2}$ The Customs-Trade Partnership Against Terrorism (C-TPAT) is a voluntary supply chain security program led by U.S. Customs and Border Protection.
} 
related work of our research are presented. Section 3 illustrates the proposed model by explaining its formal definitions with an example. Then, in section 4 the design guidelines of the model are presented. Finally, section 5 draws the conclusions and discusses our future work.

\section{Background and Related Work}

Comprehensive analysis of agent systems has shown that different design approaches are appropriate for different domain characteristics [5]. In particular, multi-agent organization frameworks are suitable to model complex environments where many independent entities coexist within explicit normative and organizational structures. Numbers of MAS methodologies with a clear organizational vision have been developed such as Gaia [6], Tropos [7], MOISE+ [8], AGR [9], INGENIAS [10], etc., which provide potential approaches for modeling inter-organizational interactions between business and governmental organizations.

All these approaches adopt the notion of role enacted by agents. Role is defined as functions and/or responsibilities that guide individual behaviors and regulate group interactions [11]. Strijbos et al. [12] discern three levels of the role concept: micro (role as task), meso (role as pattern) and macro (role as stance). Dahchour et al. [13] present a generic role model in which both static and dynamic aspects of the role relationship are considered. There is a different perspective in the field of Role-based Access Control (RBAC), in which roles are used to identify use classes for systems.

As discussed in the Introduction, context-aware applications are appropriate for modeling different interactive environments. In the field of computer science, context is regarded as a set of attributes associated to specific situations. From the perspective of cognitive modeling, a dynamic theory of context considers context as the set of all entities that influence human cognitive behavior on a specific occasion [14]. In the field of sociology, context is regarded as networks of interacting entities and focuses on the structural properties deriving from recurrent interactions among entities. In international trade, we define context as a set of states associated to interactive entities in specific environments. The regulation of business activities by governments is different according to the context of operation. An example of self-regulation context in international trade is the Authorized Economic Operator (AEO) program [15]. In this paper, we use this specific case of AEO to analyze and compare the two scenarios of direct control and self-regulation by the proposed model.

Based on the studies of MAS, Role and Context, we propose a context-aware interorganizational collaboration model which (1) gives an elaborated analysis of roles, (2) explicitly considers the influence of contexts on organizational interactions, and (3) supports a contextual refinement process for modeling organizations. We use the OperA framework as a basis for our work because firstly it is a formal organization model, secondly it has a well-defined structure for roles and dependencies, and lastly it has a software analysis tool that can be used for evaluation.

The OperA framework [4] consists of three interrelated models: The Organizational Model (OM) is the result of the observation and analysis of the domain and describes the desired behavior of the organization. The Social Model (SM) maps organizational roles to agents and describes agreements concerning the role enactment in social contracts. Roles are typically declarative entities meant to 
represent a part of the organization's design and can be taken up by the agents enacting the role. Objectives of an organization are achieved through the actions of agents. Finally, the Interaction Model (IM) specifies the interaction agreements between role-enacting agents. This paper focuses on the social structure in the OM dimension of OperA which describes organizational interactions from the designer's perspective and is also the first step in the methodology of OperA framework.

\section{A Context-Aware Inter-organizational Collaboration Model}

In this section, we introduce our model and illustrate it by an example. In the example, we apply the proposed model to describe two scenarios of direct control and self-regulation using the specific case of AEO in international trade.

\subsection{Fundamental Concepts}

We first illustrate several concepts extended from OperA, which provide a basis to the proposed model. We only show a part of the properties of each concept to keep it simplified. Note that an element presented by a lowercase letter is a member of the set indicated by the corresponding capital letter, e.g., $r \in R$.

1) Role $(r)$

A role is a set of objectives $O b j$. Objectives of a role indicate its individual responsibility, i.e., if a role is enacted, its individual responsibility is undertaken. To facilitate multiple levels of modeling from abstract to concrete, we define two kinds of roles in OperA+.

(1) Atomic role $\left(r_{A}=(\right.$ name,$\left.O b j)\right)$

Atomic roles are roles as stance which hold relatively general objectives. They provide a macro-level understanding of what tasks will be carried out.

(2) Composite role $\left(r_{C}=(\right.$ name,$O b j$, intl $\left.)\right)$

Composite roles are roles as pattern which not only express the stance through their objectives but also give more details on how to accomplish the objectives through lower level organizations, each of which is indicated by an inter-level link intl $=(r$, org $)$. The pattern is represented by the sub-roles in the lower-level organization.

2) Organization $($ org $=($ name $, R, D e p))$

An organization is a set of connected roles. Roles in an organization connect with each other through a set of role dependencies Dep which promotes group cohesion. dep $=\left(r_{1}, r_{2}, o b j\right)$ indicates that $r_{l}$ depends on $r_{2}$ for objective obj. Moreover, there is only one top level organization marked as $\operatorname{org}_{0}$ in each model and all the other organizations are derived from composite roles.

\subsection{Contextual Refinement Modeling Process}

In order to provide actors in international trade with an evolutionary understanding of their responsibilities, our proposal illustrates a modeling process for organizational interactions from an abstract level to a concrete level as depicted in Fig. 1.

First, a general specification is constructed to express the common objectives of inter-organizational collaborations in an abstract way, which captures the root goals 
of an international trade from a comprehensive perspective. For example, whether an international trade is exporting food or clothes, the general specification contains the same set of roles at an abstract level, such as exporter, carrier, customs, etc. General specifications only consist of atomic roles which give stances of how interactions are organized. Then, according to different contexts, the general specification is contextualized into different contextual specifications which describe the interactive relationships in a more detailed way and present the differences between different situations of an international trade. Contextualization applies contextual information to general specifications and transforms some of the atomic roles to composite roles which contain more information or constraints on how to realize the objectives of the roles. Finally, the whole set of contextual specifications is transformed into different operational specifications which depict complete pictures of an inter-organizational collaboration model in different executable situations. That is, actors in international trades will match their status with the contexts in contextual specifications and select corresponding roles. We can see that the three phases form a contextual refinement modeling process which gives each actor a better understanding of its individual part as well as its interactive parts. For detailed illustrations, we give the following definitions.

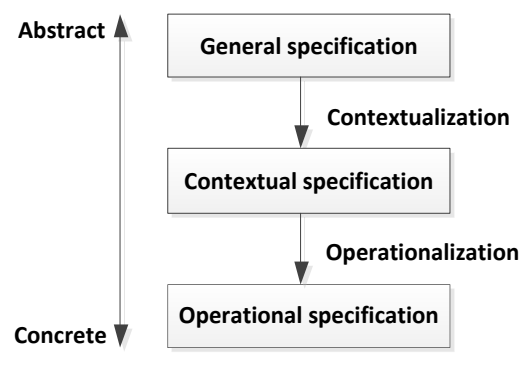

Fig. 1. Three phases of the modeling process. This figure illustrates the three phases to model organizational interactions from an abstract level to a concrete level in our proposal.

\subsubsection{General Specification}

To capture the vision of the organizational goals from the designer's perspective, we first formalize the concept of a general specification.

Definition 1. (general specification). A general specification $\operatorname{org}_{i}^{g s}, i \in N$ of an organization $\operatorname{org}_{i}$ is a tuple (name, $R_{A}$, Dep) such that:

- name is an identifier,

- $R_{A}$ is a set of roles,

- $\forall r \in R_{A}$ is an atomic role and

- Dep is a set of role dependencies. 
A general specification shows a map of abstract expectations for the collaboration model without providing detailed information or constraints on how to accomplish the objectives of each role.

To illustrate our proposal, we use a running example to explain how Regulatory authorities and Private enterprise collaborate with each other in two scenarios, viz. those of direct control and self-regulation in international trade. Table 1 shows the general specification $\operatorname{org}_{0}^{g s}$ of the top-level organization $\operatorname{org}_{0}$ in the example.

Table 1. Role table for the top level organization. The table explains the roles, their objectives and dependencies in the top level organization.

\begin{tabular}{llll}
\hline Organization & Role & Role objective & Role dependencies \\
\hline $\operatorname{org}_{0}$ & Regulatory authorities $(R a)$ & Efficient regulation & $P e$ \\
& Private enterprise $(P e)$ & Efficient action & $R a$ \\
\hline
\end{tabular}

The objectives of the $R a$ and $P e$ in $\mathrm{Org}_{0}$ are intellectual attitudes of the designer's expectations and little information is given on how to reach the objectives. Therefore, at this level, role enactors have the freedom to decide on how to perform their tasks. Furthermore, the two roles are inter-dependent for their objectives.

\subsubsection{Contextual Specification}

In international trade, organizational interactions are not only determined by individual roles or organizations but also dependent on the environment. Therefore, the notion of a context in our model is defined as the environment of a general specification that satisfies a set of given conditions, which is as follows.

Definition 2. (context). A context $c t x$ associated with a general specification $\operatorname{org}_{i}^{g s}$ is a tuple (name, $\operatorname{org}_{i}^{g s}$, State) such that:

- name is an identifier,

- $\operatorname{org}_{i}^{g s}$ indicates the contextualized organization,

- State is a set of states related to the roles in $\operatorname{org}_{i}^{g s}$,

- $\forall$ state $\in$ State $:$ state $=\left(R_{S}\right.$, Cond $), R_{S} \subseteq R_{A}\left(\operatorname{org}_{i}^{g s}\right)$ and

- Cond is a set of conditions which define the state of the roles.

State defines the context for the general specification $\operatorname{org}_{i}^{g s}$.

$\forall$ state $\in$ State $:$ state $=\left(R_{S}\right.$, Cond $), R_{S} \subseteq R_{A}\left(\operatorname{org}_{i}^{g s}\right)$ indicates the inter-state between multiple roles. For example, (exporter, carrier, long-term) indicates that the exporter and the carrier interact with each other in the context of long-term cooperation. Specifically, when there is only one role in $R_{S}$, the intra-state of an individual role is specified.

In the example, we use a specific case of AEO certification to illustrate the notion of context. With an AEO certification, a company is trusted throughout the EU for customs related regulations and is granted the power of self-control [16]. Therefore, in our example, with AEO corresponds to the scenario of self-regulation while without AEO corresponds to the scenario of direct control. Based on these two scenarios of direct control and self-regulation, we define two contexts below. 
$\left(c_{1}{ }_{1}, \operatorname{org}_{0}^{g s},\{(R a, P e\right.$, without an AEO certification $\left.)\}\right)$,

$\left(\operatorname{ctx}_{2}, \operatorname{org}_{0}^{g s},\{(R a, P e\right.$, with an AEO certification $\left.)\}\right)$.

The influence of a context on an organization is realized through role transformation from atomic role to composite role. In this sense, context adds more information to some of the roles by extending them to lower level organizations, which realizes the process of adding regulations on some of the roles in international trade. Based on the definition of context and its influence on other modeling elements, we formalize the concept of contextual specification as follows.

Definition 3. (contextual specification). A contextual specification $\operatorname{org}_{i}^{c s}$ of an organization $\operatorname{org}_{i}$ is a tuple (name, ctx $, R_{A}^{\prime}, R_{C}$ ) such that:

- name is an identifier,

- $c t x$ is a context,

- $R_{A}^{\prime}$ is a set of atomic roles,

- $R_{C}$ is a set of composite roles and

- $R_{A}^{\prime} \cap R_{C}=\phi \wedge R_{A}^{\prime} \cup R_{C}=R_{A}\left(\operatorname{org}_{i}^{g s}(\operatorname{ctx})\right)$.

$R_{C}$ specifies which roles in the general specification associated with ctx are transformed from atomic roles to composite roles. This is in accordance with the fact that different contexts have different influence on different sets of roles. $R_{A}^{\prime} \cap R_{C}=\phi \wedge R_{A}^{\prime} \cup R_{C}=R_{A}\left(\operatorname{org}_{i}^{g s}(c t x)\right)$ indicates that all the roles either atomic or composite in contextual specifications are derived from the roles in a general specification. Besides, a role can be influenced by multiple contexts in which it is extended to different lower level organizations of sub-roles and dependencies.

Given $\operatorname{org}_{0}^{g s}$, there are two contexts with different sets of $R_{C}$. Therefore, two contextual specifications are constructed.

$$
\left(\operatorname{org}_{0}^{c s_{1}}, c t x_{1},\{P e\}, R(R a)\right) \text { and }\left(\operatorname{org}_{0}^{c s_{2}}, c t x_{2}, \phi, R(R a) \cup R(P e)\right) \text {. }
$$

$\operatorname{org}_{0}^{c s_{1}}$ shows the scenario of direct control described by $c t x_{1}$ in which the $P e$ is fully regulated by the $R a$. $\operatorname{org}_{0}^{c s_{2}}$ shows the scenario of self-regulation described by $c t x_{2}$ in which the $P e$ undertakes a part of the responsibilities from the $R a$. Detailed descriptions are shown respectively in Table 2 and 3. Note that we use the same name for the atomic role in the general specification, its corresponding composite role in the contextual specification and its referred lower level organization.

In $c t x_{1}$, only the $R a$ transforms to a composite role which refers to a lower level organization consisting of five atomic roles that are fine-grained divisions of the $R a$. This is the situation of direct control in international trade and the $R a$ is extended to restrict the behaviors of its enactors. That is, role enactors of the $R a$ should follow the pattern constituted by the lower level organization. Therefore, at this level, as the context brings more information from the environment, detailed specification of the organizational interactions should be specified. 
Table 2. Role table for the lower level organization in context $c t x_{1}$. The table explains the roles, their objectives and dependencies in the lower level organization of $R a$ in $c t x_{1}$.

\begin{tabular}{llll}
\hline Organization & Role & Role objective & Role dependencies \\
\hline$R a$ & Norm maker & Effective norms making & \\
& Norm specifier & Specify valuable norms & Norm maker \\
& Control indicator & Make efficient control & Norm specifier \\
& maker & indicators & Efficient action monitoring \\
& Action monitor & $\begin{array}{l}\text { Control indicator } \\
\text { maker }\end{array}$ \\
& Sanctioner & Correct sanctioning & Action monitor \\
\hline
\end{tabular}

Table 3. Role table for the lower level organizations in context $c t x_{2}$. The table explains the roles, their objectives and dependencies in the lower level organizations of $R a$ and $P e$ in $c t x_{2}$.

\begin{tabular}{llll}
\hline Organization & Role & Role objective & Role dependencies \\
\hline$R a$ & Norm maker & Make effective norms & \\
& Control monitor & Efficient control monitoring & Norm maker \\
& Sanctioner & Correct sanctioning & Control monitor \\
& Norm specifier & Specify valuable norms & \\
& Control indicator & Make efficient control & Norm specifier \\
& maker & indicators & Cfficient action monitoring \\
& Action monitor & Control indicator \\
& & & maker \\
\hline
\end{tabular}

In $c t x_{2}$, both the $R a$ and the $P e$ transform to a composite role. It can be seen that some of the roles in the lower level organization of $R a$ in $c_{t} x_{1}$ shift to the lower level organization of $\mathrm{Pe}$ in $\mathrm{ctx}_{2}$. This is the situation of self-control in international trade in which a part of the responsibilities of the $R a$ transfers to the $P e$ with AEO certification. We can see that the same general specification transforms to two contextual specifications with different extensions or restrictions on how to reach the collective goals of the top level organizations in a detailed way. This is an intuitive way to explain how the organizational interactions are evolved.

\subsubsection{Operational Specification}

A general specification has multiple extensions because of different contexts. That is, the high level abstraction can be re-used in different situations by applying different contexts. However, at runtime, there must be a complete specification which describes the model in a whole. Therefore, we give the definition of an operational specification below.

Definition 4. (operational specification). An operational specification os of an interorganizational collaboration is a tuple (name, $\mathrm{Org}^{c s}$, $\operatorname{org}_{0}^{c s}, R^{*}$, Intl $^{*}$ ) such that:

- name is an identifier,

- $\operatorname{Org}^{c s}$ is a set of contextual specifications,

- $\forall \operatorname{org}_{i_{1}}^{c s}, \operatorname{org}_{i_{2}}^{c s} \in \operatorname{Org}^{c s}, i_{1} \neq i_{2}: \operatorname{org}_{i}^{g s}\left(\operatorname{ctx}\left(\operatorname{org}_{i_{1}}^{c s}\right)\right) \neq \operatorname{org}_{i}^{g s}\left(\operatorname{ctx}\left(\operatorname{org}_{i_{2}}^{c s}\right)\right)$, 
- $\operatorname{org}_{0}^{c s} \in \mathrm{Org}^{c s}$ is a contextual specification of the top level organization,

- $R^{*}=\bigcup_{o r g}^{c s} \in \operatorname{Org}^{c s}\left(R_{A}^{\prime}\left(\operatorname{org}_{i}^{c s}\right) \bigcup R_{C}\left(\operatorname{org}_{i}^{c s}\right)\right)$ is the set of all roles,

- Intl ${ }^{*}: \bigcup_{\operatorname{org}_{i}^{c s} \in \operatorname{Org}_{\mathrm{g}}^{c s}} R_{C}\left(\operatorname{org}_{i}^{c s}\right) \rightarrow \operatorname{Org}^{c s} \backslash \operatorname{org}_{0}^{c s}$ is a bijective function which maps each composite role onto a lower level organization, and

- $\forall$ org $\in \operatorname{Org}^{c s} \backslash \operatorname{org}_{0}^{c s}: \exists !$ intl $\in \operatorname{Intl}^{*}:$ org $($ intl $)=$ org .

$\forall \operatorname{org}_{i_{1}}^{c s}, \operatorname{org}_{i_{2}}^{c s} \in \operatorname{Org}^{c s}, i_{1} \neq i_{2}: \operatorname{org}_{i}^{g s}\left(\operatorname{ctx}\left(\operatorname{org}_{i_{1}}^{c s}\right)\right) \neq \operatorname{org}_{i}^{g s}\left(\operatorname{ctx}\left(\operatorname{org}_{i_{2}}^{c s}\right)\right)$ indicates that in an operational specification there can't be two contexts associated with the same general specification. $\forall$ org $\in \operatorname{Org}^{c s} \backslash \operatorname{org}_{0}^{c s}: \exists !$ intl $\in \operatorname{Intl}^{*}:$ org $($ intl $)=$ org indicates that except the top level organization, all other organizations have only one inward inter-level link so that no loop exists in operational specifications. An operational specification is a hierarchy in which the top level organization forms its root while lower level organizations form its inner nodes and leaves. The set of all contexts in an operational specification builds up the runtime environment.

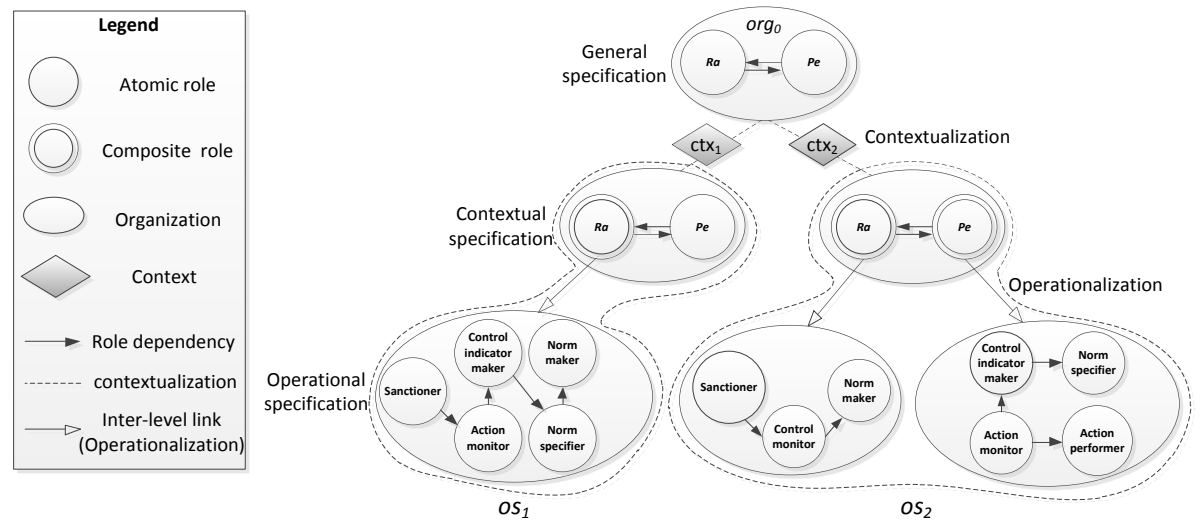

Fig. 2. Modeling process of the example. This figure illustrates the legend of the proposed model and how the two operational specifications are derived from the general specification.

Fig. 2 shows the two operational specifications $o s_{1}$ and $o s_{2}$ circled by dashed lines. They are respectively derived from $\operatorname{org}_{0}^{c s_{1}}$ and $\mathrm{org}_{0}^{c s_{2}}$. Each operational specification contains a complete description of organizational interactions associated with its context, which is an executable specification that can be seen as the assembling processes of different agents. For example, a company with an AEO certification in the Netherlands exports goods to another country in the EU and the Dutch Customs has to perform regulations on it. In this case, the company and the Dutch Customs fit in with the interactive environment of $c t x_{2}$, which indicates that each of them has to perform as the specifications of the lower level organizations in $o s_{2}$. 


\section{Design Guidelines}

In order to illustrate how contexts influence specifications, we give the design guidelines of the proposed model continued with the example which is shown in Fig. 2 as a tree-like structure.

At the top, the root of the tree is a general specification of the top level organization which is made up of atomic roles. That is, in international trade, regulative authorities and private enterprises have the same interactive relationships at an abstract level. Contextualization is depicted as dashed lines and the root of the tree is extended to different contextual specifications through transforming some of the atomic roles into composite roles. It can be seen that a general specification has multiple dashed lines connected with it, which indicates that there can be multiple contexts related to one general specification. Contextualization is the process of detailing information on how to realize the objectives of a role in a specific circumstance, which provides an adapted way of generating concrete regulations according to different situations in international trade. Among the different contexts of contextualization connected with the same general specification, they have different effects on the general specification. Contextual specifications are differentiated by the extensions of their composite roles, i.e., the referred lower level organizations. Those lower level organizations are the concrete information or regulation on how to accomplish the objectives of the composite roles in a specific context. Operationalization is the process of selecting and assembling among all the alternative contextual specifications during run time according to the real interactive environment, which generates an operational specification that illustrates the whole executive environment and how the global objectives are achieved in terms of finergrained organizations and roles. Moreover, those lower level organizations in the contextual specifications can again be contextualized according to their own contexts, which facilitates a recursive modeling process.

Organizations usually have multiple contextual specifications, which is in accordance with the fact that business and governmental organizations have different collaborative relationships under different circumstances. However, for each situation, only one of the contextual specifications with the same general specification can be instantiated, i.e., only one dashed line (contextualization) connected with the same general specification is selected. Therefore, each operational specification contains a unique set of contextual specifications with different general specifications from an abstract level to a concrete level.

Interactions between business and governmental organizations are subject to a large number of norms and regulations [17]. Norms of regulative issues are very complex and are to a large extent only implicitly represented by governmental organizations. Most of the knowledge is only in the heads of the government experts. Therefore, there are two difficulties with respect to the communications between business and governmental organizations: (1) Business organizations have to elicitate themselves the norms from the governmental organizations, (2) The norms have to be customized to the specifics of each business organization, e.g., safety risks for a dairy company is primarily food-safety, whereas safety risks for a scrap metal trading company is hazardous waste, or even hidden bombs, as bombs can be easily hidden in scrap metal. To this end, our proposal can help to solve these two difficulties: (1) by 
structuring the contextual refinement modeling process to guide both business organizations and governmental organizations to build their interactions from an abstract level to a concrete level, which provides both of them a better understanding of their responsibilities, and (2) by using contexts to differentiate the communications between business organizations and governmental organizations according to their status, i.e., business type, regulation policy, etc. Thus, the interaction process between business and governmental organizations can be viewed as a norm negotiation process in a multi-agent community, where agents can communicate with each other to determine their contextual norms through lower level specifications in the proposed model. This provides a potential solution to deal with the communication problems about self-regulation between multi-agents that jointly create shared norms, i.e., business and governmental organizations that co-create an operationalization of the open norms in legislation [18].

\section{Conclusions and Future Work}

This paper proposes a context-aware inter-organizational collaboration model, which spans the model development process from abstract attitudes to concrete implementation. The three phases in the proposal are a natural reflection of an intuitive modeling procedure but with formal definitions. We have applied the model to analyze and compare the direct control and the self-regulation contexts in international trade. The framework supports users to understand their models during the procedure and makes it possible for users to reflect their design patterns even at the final operational stage.

Our current and future work includes extending the proposed model to the enactment layer which focuses on how to model enactors of roles such as business organizations, governmental organizations and individuals in the international trade environment, and obtain a good match between agents and roles in the specifications according to their characteristics. Moreover, a software platform is being developed to simulate the interactions between business and governmental organizations to find better solutions for the problem of norm negotiation between them. We will also work on modeling evolution from one to another organization form.

\section{References}

1. Chea, S., Bui, T.X.: A multi-process actors strategic analysis model for flexible E-business proposition based on value networks configuration framework. In: Proc. 10th Americas Conference on Information Systems, New York, pp. 2544-2548 (2004)

2. Yang, S.J.H., Zhang, J., Chen, I.Y.L.: A JESS-enabled context elicitation system for providing context-aware Web services. Expert System with Applications 34(4), 2254-2266 (2008)

3. Beydoun, G., Low, G., Henderson-Sellers, B., Mouratidis, H., Gomez-Sanz, J., Pavón, J., Gonzalez-Perez, C.: FAML: A generic metamodel for MAS development. IEEE Transactions on Software Engineering 35(6), 841-863 (2009)

4. Dignum, M.V.: A model for organizational interaction: based on agents, founded in logic. Doctoral Thesis. DSpace at Utrecht University (2004) 
5. Dignum, M.V., Dignum, F.: Designing agent systems: State of the practice. International Journal on Agent-Oriented Software Engineering 4(3) (2010)

6. Zambonelli, F., Jennings, N.R., Wooldridge, M.: Developing multiagent systems: the Gaia Methodology. ACM Transactions on Software Engineering and Methodology 12(3), 317370 (2003)

7. Bresciani, P., Giorgini, P., Giunchiglia, F., Mylopoulos, J., Perini, A.: Tropos: an agentoriented software development methodology. Journal of Autonomous Agent and MultiAgent Systems 8(3), 203-236 (2004)

8. Hübner, J.F., Sichman, J.S., Boissier, O.: A Model for the Structural, Functional, and Deontic Specification of Organizations in Multiagent Systems. In: Proc. 16th Brazilian Symposium on Artificial Intelligence: Advances in Artificial Intelligence, Berlin, pp. 118128 (2002)

9. Ferber, J., Gutknecht, O., Michel, F.: From agents to organizations: an organizational view of multi-agent systems. In: Giorgini, P., Müller, J.P., Odell, J.J. (eds.) AOSE 2003. LNCS, vol. 2935, pp. 214-230. Springer, Heidelberg (2004)

10. Pavón, J., Gómez-Sanz, J.J., Fuentes, R.: The INGENIAS methodology and tools. In: Henderson-Sellers, B., Giorgini, P. (eds.) Agent-oriented Methodologies, pp. 236-276. Idea Group, USA (2005)

11. Hare, A.P.: Types of roles in small groups: A bit of history and a current perspective. Small Group Research 25, 443-448 (1994)

12. Strijbos, J.W., De Laat, M.F.: Developing the role concept for computer-supported collaborative learning: An explorative synthesis. Computers in Human Behavior 26(4), 495-505 (2010)

13. Dahchour, M., Pirotte, A., Zimanyi, E.: A role model and its metaclass implementation. Information Systems 29(3), 235-270 (2004)

14. Kokinov, B.: A dynamic approach to context modeling. In: Brezillon, P., Abu-Hakima, S. (eds.) IJCAI-WS 1995, pp. 59-72 (1995)

15. Burgemeestre, B., Hulstijn, J., Tan, Y.H.: Towards an architecture for self-regulating agents: a case study in international trade. In: Proc. 2nd Multi-Agent Logics, Languages, and Organisations Federated Workshops, Italy. CEUR Workshop Proceedings, vol. 494 (2009)

16. European Commission: AEO Guidelines, http: / / ec.europa.eu/taxation_customs / customs /

17. Burgemeestre, B., Hulstijn, J., Tan, Y.H.: Value-Based argumentation for justifying compliance. In: Proc. 5th International Workshop on Deontic Logic in Computer Science, pp. 214-228. Springer, Berlin (2010)

18. Burgemeestre, B., Hulstijn, J., Tan, Y.H.: Norm emergence in regulatory compliance. In: Proc. 5th International Workshop on Normative Multi-Agent Systems, UK (2010) 